PROCEEDINGS OF THE

AMERICAN MATHEMATICAL SOCIETY

Volume 126, Number 1, January 1998, Pages 45-52

S 0002-9939(98)04230-0

\title{
QUOTIENT DIVISIBLE ABELIAN GROUPS
}

\author{
A. FOMIN AND W. WICKLESS
}

(Communicated by Ronald M. Solomon)

Dedicated to the memory of Ross A. Beaumont

\begin{abstract}
An abelian group $G$ is called quotient divisible if $G$ is of finite torsion-free rank and there exists a free subgroup $F \subset G$ such that $G / F$ is divisible. The class of quotient divisible groups contains the torsion-free finite rank quotient divisible groups introduced by Beaumont and Pierce and essentially contains the class $\mathcal{G}$ of self-small mixed groups which has recently been investigated by several authors. We construct a duality from the category of quotient divisible groups and quasi-homomorphisms to the category of torsionfree finite rank groups and quasi-homomorphisms. Our duality when restricted to torsion-free quotient divisible groups coincides with the duality of Arnold and when restricted to $\mathcal{G}$ coincides with the duality previously constructed by the authors.
\end{abstract}

All groups will be additive and abelian. The torsion-free rank of a group $G$ is the rank of $G / T(G)$, where $T(G)$ is the torsion subgroup of $G$. The $p$-rank of $G$ is the rank of $G / p G$. For a group $G$ and prime $p$ we let $G_{p}^{\wedge}$ be the $p$-adic completion of $G / p^{\omega} G$ and regard $G / p^{\omega} G \subset G_{p}$. A $\diamond$ marks the end of a proof.

Definition 1. An abelian group $G$ is quotient divisible (qd) if $G$ is of finite torsion-free rank and there exists a free subgroup $F \subset G$ with $G / F$ a divisible group.

We remark that the torsion-free qd groups are precisely the classical quotient divisible groups introduced by Beaumont and Pierce in 1961 [BP]. The following proposition is elementary.

Proposition 2. For an abelian group $G$ of finite torsion-free rank the following are equivalent:

(1) $G$ is $q d$.

(2) There exists a free subgroup $F \subset G$ such that, for each positive integer $m$, $G=F+m G$.

(3) There exists a free subgroup $F \subset G$ such that, for each prime $p, G=F+p G$.

Definition 3. For each prime $p$ let $M(p)$ be a module over the ring of $p$-adic integers $Z_{p}^{\widehat{~}}$. A subgroup $A \subset \prod_{p} M(p)$ satisfies the projection condition if there is a free subgroup of finite rank $F \subset A$ such that, for every $p$, the natural projection of $F$ into $M(p)$ generates $M(p)$ as a $Z_{p^{-}}$module.

We call a free subgroup $F$ of a group $G$ full if $G / F$ is torsion.

Received by the editors June 14, 1996.

1991 Mathematics Subject Classification. Primary 20K21, 20K40.

(C)1998 American Mathematical Society 
Theorem 4. Let $A$ be reduced of torsion-free rank $n$. Then $A$ is qd if and only if $A$ is (can be embedded as) a pure subgroup of a direct product of p-adic modules $A \subset \prod_{p} M(p)$ such that $A$ satisfies the projection condition.

Proof. Suppose $A$ is reduced qd of torsion-free rank $n$. Let $F \subset A$ be free of rank $n$ with $A / F$ divisible. By Proposition 2 , if $T_{p}$ is the $p$-torsion part of $A$, then $T_{p}$ cannot have $n+1$ nonzero cyclic summands. Hence each $T_{p}$ is finite. For each $p$ write $A=T_{p} \oplus A_{p}^{\prime}$ where $A_{p}^{\prime}$ has no $p$-torsion. The first Ulm subgroup $A^{1}=\bigcap_{p} p^{\omega} A$ is contained in $\bigcap_{p} A_{p}^{\prime}$ since an element of infinite $p$-height cannot have a nonzero $T_{p}$ component in any of the previous decompositions. We claim that for each $a \in A^{1}$ and prime $p$, there exists a $b \in A^{1}$ such that $a=p b$. Plainly, we can choose $b \in A_{p}^{\prime}$ with $a=p b$. If $q \neq p$ is prime then $b$ will have infinite $q$-height since $a$ does. For each positive integer $k$ there exists $b_{k} \in A_{p}^{\prime}$ with $p^{k+1} b_{k}=a$. Hence $p\left(b-p^{k} b_{k}\right)=0$ and, since $\left(b-p^{k} b_{k}\right) \in A_{p}^{\prime},\left(b-p^{k} b_{k}\right)=0$. Thus $b$ has infinite $p$-height so that $b \in A^{1}$. We have shown that $A^{1}$ is divisible. Since $A$ is reduced, $A^{1}=0$.

It follows that $A$ is embedded as a pure subgroup of its $Z$-adic completion $A^{\wedge}=$ $\prod_{p} M(p)$ where $M(p)=\widehat{A_{p}}$, the $p$-adic completion of $A / p^{\omega} A$. (See [Fu], Sections 39 and 40.) It remains to show that $A$ satisfies the projection condition. In our context each projection $\pi_{p}$ is the factor map $A \rightarrow A / p^{\omega} A$ followed by inclusion $A / p^{\omega} A \rightarrow M(p)$. Thus each $M(p) / \pi_{p}(A)$ is divisible. But $A / F$ is divisible so that $\pi_{p}(A) / \pi_{p}(F)$ is also divisible. Hence $M(p) / \pi_{p}(F)$ is divisible. Since $Z_{p}^{-}\left[\pi_{p}(F)\right]$ is a finitely generated submodule, hence a summand, of the $p$-adic module $M(p)$, and $M(p)$ is reduced, it follows that $M(p)=Z_{p}^{\curlyvee}\left[\pi_{p}(F)\right]$ for each prime $p$.

Conversely, suppose that $A$ is a pure subgroup of $\prod_{p} M(p)$ such that $A$ satisfies the projection condition. Let $F=\bigoplus Z x_{j} \subset A$ be a full free subgroup such that

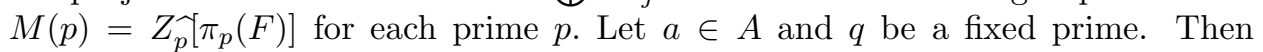
$\pi_{q}(a)=\sum \alpha_{j} \pi_{q} x_{j}$ for $q$-adic integers $\alpha_{j}$. For each $j$ let $a_{j} \in Z$ be congruent to $\alpha_{j}$ $\bmod q$. Then $a-\sum a_{j} x_{j}$ is divisible by $q$ in $\prod_{p} M(p)$, hence divisible by $q$ in $A$. We have shown that $A / F$ is $q$-divisible for each $q$ and the proof is complete.

Definition 5. Let $\mathcal{Q D}$ be the category with objects qd groups with reduced torsion part and maps quasi-homomorphisms; that is,

$$
\operatorname{Hom}_{\mathcal{Q D}}\left(A, A^{\prime}\right)=Q \otimes_{Z} \operatorname{Hom}_{Z}\left(A, A^{\prime}\right)
$$

Let $\mathcal{Q} \mathcal{D}_{0}$ be the full subcategory of $\mathcal{Q D}$ with objects torsion-free quotient divisible groups. Let $\mathcal{Q} \mathcal{T} \mathcal{F}$ be the category with objects torsion-free finite rank groups and maps quasi-homomorphisms. Let $\mathcal{Q G}$ be the category with objects self-small mixed abelian groups $G$ such that $G / T(G)$ is finite rank divisible and maps quasihomomorphisms.

The category $\mathcal{Q G}$ has been investigated by several authors ([AGW], [FiW], $[\mathrm{FoW}],[\mathrm{GW}],[\mathrm{VW}],[\mathrm{Wi}])$. The relation between $\mathcal{Q G}$ and $\mathcal{Q D}$ is described in the following proposition.

Proposition 6. The category $\mathcal{Q G}$ can be identified with the full subcategory $\mathcal{Q D}_{t}$ of $\mathcal{Q D}$ whose objects $H$ satisfy the additional restriction that the p-adic modules $M(p)$ of Theorem 4 , defined with respect to $A=H / V, V$ the maximal divisible subgroup of $H$, are torsion for each $p$.

Proof. It follows from Corollary 2.4 of [AGW] that every reduced group $A$ in $\mathcal{Q G}$ is a pure subgroup of $\prod T_{p}(A)$, where $T_{p}(A)$ is the $p$-torsion subgroup of $A$, with 
the projection condition holding for some full free subgroup $F \subset A$ for almost all primes $p$. Let $S$ be the finite subset of primes such that $Z_{p}^{\wedge}\left[\pi_{p}(F)\right] \neq T_{p}(A)$. For $p \notin S, T_{p}(A)$ will be finite of rank less than or equal to rank $F=$ torsion-free rank $A$. In order for $A$ to be self-small the groups $T_{p}(A), p \in S$, also must necessarily be finite [AGW]. Thus any object $G$ of $\mathcal{Q G}$ is a direct sum $G=V \oplus A=V \oplus\left(B \oplus A^{\prime}\right)$ where $V$ is finite rank torsion-free divisible, $B=\bigoplus_{p \in S} T_{p}(A)$ is a finite group and $A^{\prime}=A \cap \prod_{p \notin S} T_{p}(A)$ is a pure subgroup of $\prod T_{p}\left(A^{\prime}\right)$ with the projection condition. By Theorem $4, A^{\prime}$ is in $\mathcal{Q D}$. Hence each object $G$ of $\mathcal{Q G}$ is a direct sum $G=B \oplus\left(V \oplus A^{\prime}\right)$ of a finite group and an object $H=V \oplus A^{\prime}$ of $\mathcal{Q D}$. Since we are working in quasi-homomorphism categories the correspondence $G \rightarrow H$ is an embedding of $\mathcal{Q G}$ as a full subcategory of $\mathcal{Q D}$.

To show that the embedded copy of $\mathcal{Q G}$ in $\mathcal{Q D}$ is exactly $\mathcal{Q D}_{t}$, let $A$ be a pure subgroup of a product of torsion $p$-adic modules $\prod_{p} M(p)$ with the projection condition. We show that, for each fixed prime $q, M(q)=T_{q}(A)$ and, hence, by Corollary 2.4 of $[\mathrm{AGW}]$, that $A$ is a group in $\mathcal{Q G}$. Let $q$ be fixed and $m \in M(q)$. There is an $f=\left(f_{p}\right) \in F$, a full free subgroup of $A$, with $f_{q}=m$. If $q^{k} M(q)=0(M(q)$ is finitely generated) then $q^{k} f \in \prod_{p \neq q} M(p) \cap A$. Since elements of $\prod_{p \neq q} M(p)$ have infinite $q$-height and $A$ is pure in $\prod_{p} M(p)$ it follows that $q^{k} f=q^{2 k} a$ for some $a \in A$. Thus $q^{k}(f-m)=q^{2 k} a=q^{k}\left(q^{k} a\right)$. Since division by $q$ in $\prod_{p \neq q} M(p)$ is unique we have $f-m=q^{k} a \in A$. Thus $m \in A$ and $M(q)$ coincides with the $q$-torsion subgroup of $A$. The proof is complete.

For the reader's convenience, we summarize constructions that have been useful in the study of torsion-free finite rank groups. For additional details see [Fo1]. Let $G$ be torsion-free of rank $n$ with free subgroup $F=\bigoplus_{i=1}^{n} Z x_{i}$. For each $p$ let $r_{p}$ be the $p$-rank of $G$. Then the torsion group $G / F$ has the form:

$$
G / F=\bigoplus_{p}[G / F]_{p}=\bigoplus_{p}\left[\bigoplus_{i=1}^{r_{p}} Z\left(p^{k_{i p}}\right) \oplus \bigoplus_{i=r_{p}+1}^{n} Z\left(p^{\infty}\right)\right], 0 \leq k_{i p}<\infty .
$$

Fix a prime $p$. For each of the first $r_{p}$ cyclic summands in (1) there is a collection of integers $a_{i j}^{p}, 1 \leq j \leq n$, such that for $y_{i}^{p}=p^{-k_{i p}} \sum_{j=1}^{n} a_{i j}^{p} x_{j}, y_{i}^{p}+F$ is a generator of that summand. If $\alpha_{i j}^{p}=a_{i j}^{p}+p^{k_{i p}} Z$ then, for $1 \leq i \leq r_{p}$, we obtain a relation $\sum_{j=1}^{n} \alpha_{i j}^{p} x_{j}=0$ in $G / p^{k_{i p}} G$. Here we regard each $G / p^{k_{i p}} G$ as a $Z / p^{k_{i p}} Z$ module in the natural way and abuse notation by writing $x_{j}$ for $x_{j}+p^{k_{i p}} G$. (If $k_{i p}=0$ we choose the zero relation $\sum_{j=1}^{n} 0 x_{j}=0$.)

For each of the $n-r_{p}$ quasi-cyclic summands in (1) we choose a set of generators $\left\{y_{i}^{p}(k)+F: 1 \leq k<\infty\right\}$ of that summand with $y_{i}^{p}(1)+F$ of order $p$ and, for $k \geq 2$, $p\left[y_{i}^{p}(k)+F\right]=y_{i}^{p}(k-1)+F$. Exactly as in the previous paragraph, each $y_{i}^{p}(k)$ determines a relation $\sum_{j=1}^{n} \alpha_{i j}^{p}(k) x_{j}=0$ in $G / p^{k} G$. For each fixed $j$ the sequence $\left\langle\alpha_{i j}^{p}(k)\right\rangle_{k}$ determines a $p$-adic integer $\alpha_{i j}^{p}$. We obtain, for each $i$ with $r_{p}<i \leq n$, a $p$-adic relation $\sum_{j=1}^{n} \alpha_{i j}^{p} x_{j}=0$ in $G_{p}$. For $p$-adic relations we continue our abuse of notation by simply writing $x_{j}$ for $\left(x_{j}+p^{\omega} G\right) \in G / p^{\omega} G \subset G_{p}$. Note that if $r_{p}=0$ for some $p$, then $[G / F]_{p}$ is divisible. In this case we obtain no finite $p$-relations,

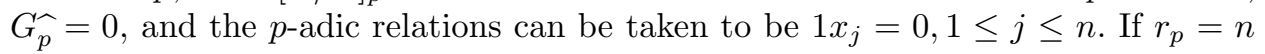
then $[G / F]_{p}$ has no quasi-cyclic summands and we obtain no $p$-adic relations.

For each $p$, we write this set of $p$-relations in matrix form: $M_{G}^{p} X=0$, where $X$ is the $n \times 1$ column vector with entries $x_{1}, \ldots, x_{n}$ and, for each $p, M_{G}^{p}$ is an $n \times n$ 
matrix with the $i$-th row consisting of elements of the ring $Z / p^{k_{i p}} Z$ for $i \leq r_{p}$ and elements of the ring $Z_{p}$ for $r_{p}<i \leq n$. In this way, to each group $G$ and fixed full free subgroup $F$, we associate a set of matrices $\left\{M_{G}^{p}\right\}$.

Conversely, given a set of $n \times n$ matrices $\left\{M^{p}\right\}$ indexed by the primes such each row of each $M^{p}$ consists of elements of the same ring, either a residue class ring $Z / p^{k_{i p}} Z$ or $Z_{p}$, we can invert our construction to obtain a torsion-free group of rank $n$. The group is constructed by starting with an abstract free group $F=\bigoplus_{i=1}^{n} Z u_{i}$ and adjoining generators corresponding to the relations $M^{p} X=0$. For any $G$, the group determined by the collection of matrices $\left\{M_{G}^{p}\right\}$ will be exactly $G$. In the proof of our main theorem we will explicitly detail the inverse construction.

We first prove two lemmas that will be needed in the proof of our main theorem.

Lemma 7. Let $G$ and $H$ be torsion-free finite rank groups, $\left\{x_{1}, \ldots, x_{n}\right\}$ a maximal linearly independent set in $G$ and $\left\{y_{1}, \ldots, y_{n}\right\}$ an arbitrary set of elements of $H$. Then there exists a homomorphism $f: G \rightarrow H$ with $f\left(x_{i}\right)=y_{i}, 1 \leq i \leq n$, if and only if $M_{G}^{p}\left(\begin{array}{l}y_{1} \\ . . \\ y_{n}\end{array}\right)=\left(\begin{array}{l}0 \\ . . \\ 0\end{array}\right)$ for all primes $p$.

Proof. Let $f$ be the mapping from the divisible hull of $G$ to the divisible hull of $H$ defined by $f\left(x_{i}\right)=y_{i}, 1 \leq i \leq n$. The requirement that all generators of $G$ will be carried by $f$ into $H$ is equivalent to the requirement that all of the above matrix equalities hold.

Definition 8. For $0 \leq k<\infty$, a $p^{k}$-relation of a torsion-free finite rank group $G$ with maximal independent set $\left\{x_{1}, \ldots, x_{n}\right\}$ is an element $\left(\alpha_{1}, \ldots, \alpha_{n}\right) \in\left(Z / p^{k} Z\right)^{n}$ such that $\sum_{i=1}^{n} \alpha_{i} x_{i}=0$ in $G / p^{k} G$. A $p^{\infty}$-relation of $G$ is an element $\left(\alpha_{1}, \ldots, \alpha_{n}\right) \in$ $\left(Z_{p}^{\gamma}\right)^{n}$ such that $\sum_{i=1}^{n} \alpha_{i} x_{i}=0$ holds in $G_{p}^{\wedge}$. (We continue to write $x_{i}$ for the appropriate coset of $x_{i}$.)

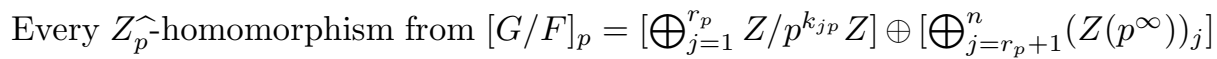
to $Z / p^{k} Z, 0 \leq k<\infty$, or to $Z\left(p^{\infty}\right)$ can be represented by a row of homomorphisms $\varphi=\left(\varphi_{1}, \ldots, \varphi_{n}\right)$. We can write the elements $a$ of

$$
\left[\bigoplus_{j=1}^{r_{p}} Z / p^{k_{j p}} Z\right] \oplus\left[\bigoplus_{j=r_{p}+1}^{n}\left(Z\left(p^{\infty}\right)\right)_{j}\right]
$$

as $n \times 1$ column matrices and thereby represent $\varphi(a)$ as a matrix product.

Lemma 9. With notation as above, an element $\left(\alpha_{1}, \ldots, \alpha_{n}\right)$ of $\left(Z / p^{k} Z\right)^{n}$ or $\left(Z_{p}^{-}\right)^{n}$ is a $p^{k}$-relation or a $p^{\infty}$-relation if and only if $\left(\varphi_{1}, \ldots, \varphi_{n}\right) M_{G}^{p}=\left(\alpha_{1}, \ldots, \alpha_{n}\right)$ for some $\left(\varphi_{1}, \ldots, \varphi_{n}\right)$. Furthermore $\left(\varphi_{1}, \ldots, \varphi_{n}\right) M_{G}^{p}=(0, \ldots, 0)$ only if $\left(\varphi_{1}, \ldots, \varphi_{n}\right)=$ $(0, \ldots, 0)$.

Proof. These results follow from the fact that the rows of each $M_{G}^{p}$ are the relations obtained from generators of $G / F$ which are linearly independent. For details see [Fo1], Lemma 2.

Theorem 10. The categories $\mathcal{Q D}$ and $\mathcal{Q T \mathcal { F }}$ are dual.

Proof. We construct a contravariant equivalence $d$ from $\mathcal{Q D}$ to $\mathcal{Q T} \mathcal{F}$. Let $G$ be an object of $\mathcal{Q D}$. Then $G$ is a qd group such that its maximal divisible subgroup $D$ is torsion-free. Hence $A=G / D$ is a reduced qd group which, by Theorem 4, can be 
regarded as a pure subgroup $A \subset A^{\wedge}=\prod_{p} A_{p}$ with the projection condition. Let $F=\bigoplus_{i=1}^{n} Z x_{i}$ be a full free subgroup such that $G / \bigoplus_{i=1}^{n} Z x_{i}$ is divisible. For each

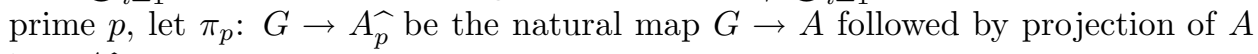
into $\widehat{A_{p}}$.

Because of the projection condition each $A_{p}$ is generated over $Z_{p}$ by the set

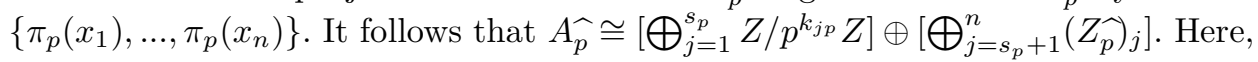
for each $p$, the $s_{p}$ are integers with $0 \leq s_{p} \leq n$ and the $k_{j p}, 1 \leq j \leq n$, are a non-decreasing sequence of nonnegative integers. For each fixed $i$ we have $\pi_{p}\left(x_{i}\right)=$ $\left(\alpha_{i 1}, \ldots, \alpha_{i n}\right)$ where the first $s_{p}$ entries are in the appropriate residue class rings $Z / p^{k_{j p}} Z$ and the remaining entries are $p$-adic integers We obtain, for each $p$, an $n \times n$ matrix $M_{p}^{G}=M\left(p, G,\left\{x_{1}, \ldots, x_{n}\right\}\right)$ whose $i$-th row is $\pi_{p}\left(x_{i}\right)$. For $j \leq s_{p}$ the $j$-th column of $M_{p}^{G}$ will contain elements of the $\operatorname{ring} Z / p^{k_{j p}} Z$. The remaining columns of $M_{p}^{G}$ will contain $p$-adic integers. Of course, just as in the construction of $M_{G}^{p}$, we allow the possibilities $s_{p}=0$ ( $A_{p}$ has no finite part $)$ and $s_{p}=n$ ( $A_{p}$ has no torsion-free part) as well as the possibility that some or all $k_{j p}=0$.

We first construct the object $d G$. Let $Q G$ be the divisible hull of $G / T(G)$. We identify $Q G$ with $\bigoplus_{i=1}^{n} Q x_{i}$. Let $\left\{x_{1}^{*}, \ldots, x_{n}^{*}\right\}$ be the basis of $Q G^{*}=\operatorname{Hom}(Q G, Q)$ dual to $\left\{x_{1}, \ldots, x_{n}\right\}$. Then $d G$ is the subgroup of $\operatorname{Hom}(Q G, Q)$ that is generated by $\bigoplus_{i=1}^{n} Z x_{i}^{*}$ and the following set. For each prime $p$ and $1 \leq j \leq s_{p}$ we include the generator $\left(a_{1 j} x_{1}^{*}+\ldots+a_{n j} x_{n}^{*}\right) / p^{k_{j p}}$. Here the $a_{i j}$ are integers such that $\alpha_{i j}=a_{i j}+$ $p^{k_{j p}} Z, 1 \leq i \leq n$, is the $j$-th column of $M_{p}^{G}$. For each $p$-adic column $\left(\alpha_{1 j}, \ldots, \alpha_{n j}\right)$ of $M_{p}^{G}\left(j>s_{p}\right)$ and for each positive integer $k$, we include a generator $\left(a_{1 j}(k) x_{1}^{*}+\right.$ $\left.\ldots+a_{n j}(k) x_{n}^{*}\right) / p^{k}$ where $a_{i j}(k)$ is an integer congruent to $\alpha_{i j}$ modulo $p^{k}$.

Next, let $G, H \in \mathcal{Q D}$ and $f: G \rightarrow H$ be a quasi-homomorphism. In particular $f$ can be regarded as a map from $Q G$ to $Q H$. The standard dual transformation $f^{*}=H o m(f, Q)$ maps $Q H^{*}$ to $Q G^{*}$. Put $d f=\left.f^{*}\right|_{d H}$.

We prove that $d f$ is a quasi-homomorphism from $d H$ to $d G$. Let $\left\{y_{1}, \ldots, y_{m}\right\}$ be the maximal independent subset of $H$ used in the construction of $d H$ and let $L$ be the $n \times m$ rational matrix defined by the equation $\left(\begin{array}{l}f\left(x_{1}\right) \\ \cdots \\ f\left(x_{n}\right)\end{array}\right)=L\left(\begin{array}{l}y_{1} \\ \cdot \cdot \\ y_{m}\end{array}\right)$. If $g \in G$ has coordinate row $\left(q_{1}, \ldots, q_{n}\right)$ with respect to $\left\{x_{1}, \ldots, x_{n}\right\}$ then $f(g)$ has coordinate row $\left(q_{1}, \ldots, q_{n}\right) L$ with respect to $\left\{y_{1}, \ldots, y_{m}\right\}$.

By replacing $f$ with a suitable integral multiple of $f$ we can assume that $f$ is a homomorphism and that $L$ has integral entries. We show that for this new $f$, $d f$ will be a homomorphism from $d H$ to $d G$. Let $B$ be $H$ factored by its maximal divisible subgroup. Then $f$ induces a homomorphism from $A$ to $B$ and hence a

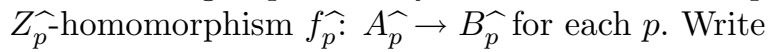

$$
\begin{aligned}
& \widehat{A_{p}}=\left[\bigoplus_{j=1}^{s_{p}}\left(Z / p^{k_{j p}} Z\right) e_{j}\right] \oplus\left[\bigoplus_{j=s_{p}+1}^{n}\left(Z_{p}^{\curlyvee}\right) e_{j}\right], \\
& \widehat{B_{p}}=\left[\bigoplus_{j=1}^{s_{p}^{\prime}}\left(Z / p^{k_{j p}^{\prime}} Z\right) e_{j}^{\prime}\right] \oplus\left[\bigoplus_{j=s_{p}^{\prime}+1}^{m}\left(Z_{p}^{\gamma}\right) e_{j}^{\prime}\right] .
\end{aligned}
$$

Here, for $1 \leq j \leq s_{p}\left(\operatorname{resp} .1 \leq j \leq s_{p}^{\prime}\right)$, order $e_{j}=p^{k_{j p}}$ (resp. order $e_{j}^{\prime}=p^{k_{j p}^{\prime}}$ ), and the $e_{j}$ (resp. $e_{j}^{\prime}$ ) are of infinite order for $j>s_{p}$ (resp. $j>s_{p}^{\prime}$ ). Note that some or 
all of the $e_{j}$ or $e_{j}^{\prime}$ and hence the accompanying coefficient ring $Z / p^{k_{j p}} Z$ or $Z / p^{k_{j p}^{\prime}} Z$ can be zero.

Let $\Delta_{p}$ be the matrix defined by the equation $\left(\begin{array}{l}f_{p}^{\mathcal{(}}\left(e_{1}\right) \\ \cdots \\ f_{p}^{\wedge}\left(e_{n}\right)\end{array}\right)=\Delta_{p}\left(\begin{array}{c}e_{1}^{\prime} \\ \cdots \\ e_{m}^{\prime}\end{array}\right)$. If $a \in A_{p}$ has coordinate row $\left(\alpha_{1}, \ldots, \alpha_{n}\right)$ with respect to the decomposition of $A_{p}$ then $f_{p}^{\sim}(a)$ has coordinate row $\left(\alpha_{1}, \ldots, \alpha_{n}\right) \Delta_{p}$ with respect to the decomposition of $B_{p}$.

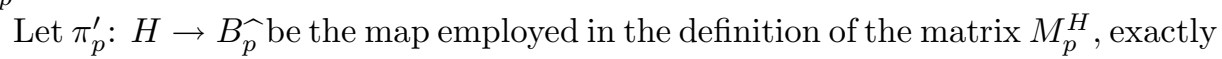
as $\pi_{p}$ was used to define $M_{p}^{G}$. Then $\pi_{p}^{\prime} f=f_{p} \pi_{p}$ for each $p$. It is a simple exercise in linear algebra to verify the matrix equalities $L M_{p}^{H}=M_{p}^{G} \Delta_{p}$. By construction of $d G,\left(x_{1}^{*}, \ldots, x_{n}^{*}\right) M_{p}^{G}=(0, \ldots, 0)$ for each prime $p$. It follows that $\left(x_{1}^{*}, \ldots, x_{n}^{*}\right) L M_{p}^{H}=$ $(0, \ldots, 0)$ for each $p$. Since $f^{*}$ is the dual of $f,\left(\begin{array}{l}f^{*}\left(y_{1}^{*}\right) \\ \ldots \\ f^{*}\left(y_{m}^{*}\right)\end{array}\right)=L^{t}\left(\begin{array}{l}x_{1}^{*} \\ \cdots \\ x_{n}^{*}\end{array}\right)$ where $L^{t}$ is the transpose of $L$. Transposing this matrix equation gives $\left(f^{*}\left(y_{1}^{*}\right), \ldots, f^{*}\left(y_{m}^{*}\right)\right)=$ $\left(x_{1}^{*}, \ldots, x_{n}^{*}\right) L$. Thus $\left(f^{*}\left(y_{1}^{*}\right), \ldots, f^{*}\left(y_{m}^{*}\right)\right) M_{p}^{H}=(0, \ldots, 0)$ for each $p$. By construction of $d H$ the matrix $M_{p}^{H}$ is the transpose of the matrix $M_{d H}^{p}$. Thus, we can appply Lemma 7 to conclude that $f^{*}$ restricted to $d H$ is a homomorphism into $d G$. We have shown that $d f$ is a homomorphism from $d H$ to $d G$, as desired.

Let $G$ be a torsion-free finite rank group with maximal independent set $\left\{x_{1}, \ldots, x_{n}\right\}$. To construct the inverse functor $d^{\prime}$ for $d$ we start with the associated set of matrices $\left\{M_{G}^{p}\right\}$. Then we construct a quotient divisible group $d^{\prime} G$ such that the divisible hull of $d^{\prime} G / T\left(d^{\prime} G\right)$ is equal to $\operatorname{Hom}(Q G, Q)$ and such that $M_{p}^{d^{\prime} G}$ is equal to the transpose of the matrix $M_{G}^{p}$ for each $p$.

More precisely, given $G$ and $\left\{x_{1}, \ldots, x_{n}\right\}$ consider the columns of the matrix $M_{G}^{p}$. Each of these columns can be regarded as an element of the $Z_{\hat{p}}$-module $M(p)=\left[\bigoplus_{j=1}^{r_{p}}\left(Z / p^{k_{j p}} Z\right)\right] \oplus\left[\bigoplus_{j=r_{p}+1}^{n}\left(Z_{p}\right)_{j}\right]$. If $M(p)$ is not generated over $Z_{p}^{\widehat{c}}$ by the columns of $M_{G}^{p}$, we can obtain a nonzero $Z_{p}$-module homomorphism $\varphi=$ $\left(\varphi_{1}, \ldots, \varphi_{n}\right): M(p) \rightarrow Z / p Z$ mapping all the columns to zero. But $\left(\varphi_{1}, \ldots, \varphi_{n}\right) M_{G}^{p}$ $=(0, \ldots, 0)$, contradicting Lemma 9 . For $1 \leq i \leq n$, let $v_{i}=\left(v_{i p}\right)$ be the element of $\prod_{p} M(p)$ such that $v_{i p}$ is the $i$-th column of $\bar{M}_{G}^{p}$. Let $A$ be the pure subgroup of $\prod_{p} M(p)$ generated by the torsion subgroup of $\prod_{p} M(p)$ and the set $\left\{v_{1}, \ldots, v_{n}\right\}$. If $M_{G}^{p}$ is the zero matrix for all $p\left(G=\bigoplus Z x_{i}\right)$ then $A=0$.

Let $F^{*}=\bigoplus_{i=1}^{n} Z x_{i}^{*} \subset G^{*}=\operatorname{Hom}(Q G, Q)$. The assignment $x_{i}^{*} \rightarrow v_{i}, 1 \leq$ $i \leq n$, determines a map $g: \operatorname{Hom}(Q G, Q) \rightarrow Q \otimes A$. Let $d^{\prime} G=A \oplus \operatorname{ker}(g)$. Since $\left\{v_{i p}: 1 \leq i \leq n\right\}$ generates $M(p)$ over $Z_{p}$ for each $p$, then $d^{\prime} G$ satisfies the projection condition with respect to $F=\bigoplus Z x_{i}^{\prime}$, where $x_{1}^{\prime}, \ldots, x_{n}^{\prime}$ are chosen such that $x_{i}^{\prime}=v_{i}+d_{i}, d_{i} \in \operatorname{ker}(g)$. Thus $d^{\prime} G$ is an object of $\mathcal{Q D}$.

Let $f: G \rightarrow H$ be a homomorphism of torsion-free finite rank groups. We need to construct a quasi-homomorphism $d^{\prime} f: d^{\prime} H \rightarrow d^{\prime} G$. Let $f^{*}: H^{*} \rightarrow G^{*}$ be the standard dual map. Since, for any torsion-free finite rank $G$, we can identify $G^{*}$ with $Q \otimes d^{\prime} G$ via $x_{i}^{*} \rightarrow 1 \otimes x_{i}^{\prime}$, we regard $f^{*}$ as a map from $Q \otimes d^{\prime} H$ to $Q \otimes d^{\prime} G$. We put $d^{\prime} f=f^{*}$ and claim that

$$
d^{\prime} f=f^{*} \in Q \otimes \operatorname{Hom}\left(d^{\prime} H, d^{\prime} G\right) .
$$

As before, we can assume the matrix $L$ of $f$ with respect to the distinguished bases $\left\{x_{1}, \ldots, x_{n}\right\}$ for $G$ and $\left\{y_{1}, \ldots, y_{m}\right\}$ for $H$ has integral entries. By Lemma 7 
$M_{G}^{p}\left(\begin{array}{l}f\left(x_{1}\right) \\ \cdots \\ f\left(x_{n}\right)\end{array}\right)=\left(\begin{array}{l}0 \\ . \cdot \\ 0\end{array}\right)$ for all $p$. It follows that $M_{G}^{p} L\left(\begin{array}{l}y_{1} \\ . \cdot \\ y_{m}\end{array}\right)=\left(\begin{array}{l}0 \\ . . \\ 0\end{array}\right)$ for all $p$. By Lemma 9, applied to each row of $M_{G}^{p} L$, for each $p$ there exists a matrix of homomorphisms $\Delta_{p}$ with $M_{G}^{p} L=\Delta_{p} M_{H}^{p}$. It follows that the matrix $\Delta_{p}$ determines a $Z_{p}$-homomorphism $\delta_{p}: B_{p}^{\widehat{a}} \rightarrow \widehat{A_{p}}$. Here $d^{\prime} H=B \oplus \operatorname{ker}\left(g^{\prime}\right)$ has been constructed in the same manner as $d^{\prime} G=A \oplus \operatorname{ker}(g)$. The matrix equality $M_{G}^{p} L=\Delta_{p} M_{H}^{p}$ can be rewritten $\left(v_{1 p}, \ldots, v_{n p}\right) L=\left(\delta_{p}\left(w_{1 p}\right), \ldots, \delta_{p}\left(w_{m p}\right)\right)$ where $v_{i p}$ and $w_{j p}$ are the $i$-th column of $M_{G}^{p}$ and the $j$-th column of $M_{H}^{p}$, respectively.

Let $\delta=\prod_{p} \delta_{p}: \prod_{p} B_{p} \rightarrow \prod_{p} A_{p}$. Then $\left(v_{1}, \ldots, v_{n}\right) L=\left(\delta\left(w_{1}\right), \ldots, \delta\left(w_{m}\right)\right)$. Since $\left\{v_{1}, \ldots, v_{n}\right\} \subset A$ and $L$ has integral entries, $\delta\left(w_{i}\right) \in A$ for $1 \leq i \leq m$. By definition of $B$, it follows that the restriction of $\delta$ to $B$ is a homomorphism from $B$ to $A$. Let $i: Q \otimes B \rightarrow Q \otimes d^{\prime} H$ be the natural embedding and $\pi: Q \otimes d^{\prime} G \rightarrow Q \otimes A$ be projection. Then, under our identifications, $\pi f^{*} i=1 \otimes \delta$ since $\left(x_{1}^{*}, \ldots, x_{n}^{*}\right) L=$ $\left(f^{*}\left(y_{1}^{*}\right), \ldots, f^{*}\left(y_{m}^{*}\right)\right)$. Thus $\pi f^{*} i$ is a quasi-homomorphism. But then, since $A, B$ are reduced and $\operatorname{ker}(g), \operatorname{ker}\left(g^{\prime}\right)$ are divisible,

$$
f^{*}:(Q \otimes B) \oplus \operatorname{ker}\left(g^{\prime}\right) \rightarrow(Q \otimes A) \oplus \operatorname{ker}(g)
$$

is a quasi-homomorphism as well.

It is easy to check that $d$ and $d^{\prime}$ satisfy $d^{\prime} d \sim 1_{\mathcal{Q D}}$ and $d d^{\prime} \sim 1_{\mathcal{Q T} \mathcal{F} \text {. This }}$ completes the proof of the theorem.

One can check directly that $d$ restricted to the full subcategory $\mathcal{Q D}_{0}$ of torsionfree finite rank qd groups coincides with Arnold duality [A]. It is also not too difficult to check that $d$ restricted to the embedded copy $\mathcal{Q G}$ of $\mathcal{Q G}$ in $\mathcal{Q D}$ (Proposition 6) coincides with the duality between the category $\mathcal{Q G}$ and the category of locally free torsion-free finite rank groups and quasi-homomorphisms, introduced by the authors in [FoW]. It follows from the construction that $d, d^{\prime}$ preserve torsionfree rank. Since, under our identifications, both $d f$ and $d^{\prime} f$ are defined to be the classical vector space dual map $f^{*}$ it follows that the functors $d, d^{\prime}$ are additive as well.

As an alternate method of proof of our main theorem, we could have constructed a category equivalence from $\mathcal{Q D}$ to the category $\mathcal{L}$ of linear mappings to finitely presented modules over the ring of universal numbers. Then we could have applied the duality of [Fo2] from $\mathcal{L}$ to $\mathcal{Q T} \mathcal{F}$. The composite of this equivalence and duality would be the same as our duality. To make our paper reasonably self-contained, we chose to construct $d$ directly.

\section{ACKNOWLEDGMENT}

We thank the referee for his or her careful reading of the paper.

\section{REFERENCES}

[A] D. Arnold, A duality for quotient divisible abelian groups of finite rank, Pac. J. Math. 42 (1972), 11-15. MR 47:361

[AGW] U. Albrecht, H.P. Goeters and W. Wickless, The flat dimension of mixed abelian groups as E-modules, Rocky Mt. J. Math. 25(2) (1995), 569-90. MR 96f:20086

[BP] R. Beaumont and R. Pierce, Torsion-free rings, Ill. J. Math. 5 (1961), 61-98. MR 26:6212

[FiW] S. Files and W. Wickless, The Baer-Kaplansky Theorem for a class of global mixed abelian groups, Rocky Mt. J. Math. 26(2) (1996), 593-613. CMP 96:17 
[Fo1] A. Fomin, The category of quasi-homomorphisms of abelian torsion-free groups of finite rank, Cont. Math. 131 (1992), 91-111. MR 93j:20108

[Fo2] - Finitely presented modules over the ring of universal numbers, Cont. Math. 171 (1994), 109-120. MR 95i:20074

[FoW] A. Fomin and W. Wickless, Categories of mixed and torsion-free abelian groups, Abelian Groups and Modules, Kluwer, Boston, 1995, 185-92. MR 97c:20083

[Fu] L. Fuchs, Infinite Abelian Groups I, II, Academic Press, New York, 1970, 1973. MR 41:333; MR 50:2362

[GW] S. Glaz and W. Wickless, Regular and principal projective endomorphism rings of mixed abelian groups, Comm. in Algebra 22(4) (1994), 1161-76. MR 95a:20060

[VW] C. Vinsonhaler and W. Wickless, Realizations of finite dimensional algebras over the rationals, Rocky Mt. J. Math.24(4) (1994), 1553-65. MR 96e:20089

[Wi] W. Wickless, A functor from mixed groups to torsion-free groups, Cont. Math. 171 (1995), 407-19. MR 95k:20090

Algebra Department, Moscow State Pedagogical University, Moscow, Russia

E-mail address: fomin.algebra@mpgu.msk.su

Department of Mathematics, University of Connecticut, Storrs, Connecticut 06269

E-mail address: wjwick@uconnvm.uconn.edu 